\title{
Assessment of genetic diversity of Plasmodium falciparum circumsporozoite protein in Sudan: the RTS,S leading malaria vaccine candidate
}

\author{
Nouh Saad Mohamed ${ }^{1,2^{*}}$ (D, Hanadi AbdElbagi ${ }^{2}$, Ahad R. Elsadig ${ }^{3}$, Abdalla Elssir Ahmed $^{2}$, \\ Yassir Osman Mohammed ${ }^{1}$, Lubna Taj Elssir ${ }^{1}$, Mohammed-Ahmed B. Elnour ${ }^{1}$, Yousif Ali ${ }^{4}$, Mohamed S. Ali', \\ Omnia Altahir ${ }^{1}$, Mustafa Abubakr ${ }^{6}$, Emmanuel Edwar Siddig ${ }^{7}$, Ayman Ahmed ${ }^{2}$ and Rihab Ali Omer ${ }^{8}$
}

\begin{abstract}
Background: The currently used malaria vaccine, RTS,S, is designed based on the Plasmodium falciparum circumsporozoite protein (PfCSP). The pfcsp gene, besides having different polymorphic patterns, can vary between $P$. falciparum isolates due to geographical origin and host immune response. Such aspects are essential when considering the deployment of the RTS,S vaccine in a certain region. Therefore, this study assessed the genetic diversity of $P$. falciparum in Sudan based on the pfcsp gene by investigating the diversity at the N-terminal, central repeat, and the C-terminal regions.
\end{abstract}

Methods: A cross-sectional molecular study was conducted; P. falciparum isolates were collected from different health centres in Khartoum State between January and December 2019. During the study period, a total of 261 febrile patients were recruited. Malaria diagnosis was made by expert microscopists using Giemsa-stained thick and thin blood films. DNA samples were examined by the semi-nested polymerase chain reaction (PCR). Single clonal infection of the confirmed $P$. falciparum cases, were used to amplify the pfcsp gene. The amplified amplicons of pfcsp have been sequenced using the Sanger dideoxy method. The obtained sequences of pfcsp nucleotide diversity parameters including the numbers of haplotypes (Hap), haplotypes diversity (Hapd), the average number of nucleotide differences between two sequences $(p)$, and the numbers of segregating sites $(S)$ were obtained. The haplotype networks were constructed using the online tcsBU software. Natural selection theory was also tested on pfcsp using Fuand Li's D, Fuand Li's F statistics, and Tajima's D test using DnaSP.

Results: In comparison with the different pfcsp reference strains, the Sudanese isolates showed high similarity with other African isolates. The results of the N-terminal region showed the presence of 2 different haplotypes with a Hapd of $0.425 \pm 0.00727$. The presence of the unique insertion of NNNGDNGREGKDEDKRDGNN was reported. The KLKQP motif was conserved in all the studied isolates. At the central repeat region, 11 haplotypes were seen with a Hapd of $0.779 \pm 0.00097$. The analysis of the genetic diversity in the C-terminal region showed the presence of 10 haplotypes with a Hapd of $0.457 \pm 0.073$. Several non-synonymous amino acids changes were also seen at the Th2R and the Th3R

\footnotetext{
${ }^{*}$ Correspondence: nouh_saad@outlook.com

${ }^{2}$ Molecular Biology Unit, Sirius Training and Research Centre, Khartoum, Sudan

Full list of author information is available at the end of the article
}

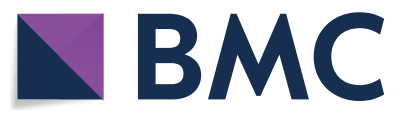

(c) The Author(s) 2021. Open Access This article is licensed under a Creative Commons Attribution 4.0 International License, which permits use, sharing, adaptation, distribution and reproduction in any medium or format, as long as you give appropriate credit to the original author(s) and the source, provide a link to the Creative Commons licence, and indicate if changes were made. The images or other third party material in this article are included in the article's Creative Commons licence, unless indicated otherwise in a credit line to the material. If material is not included in the article's Creative Commons licence and your intended use is not permitted by statutory regulation or exceeds the permitted use, you will need to obtain permission directly from the copyright holder. To view a copy of this licence, visit http://creativecommons.org/licenses/by/4.0/. The Creative Commons Public Domain Dedication waiver (http://creativeco mmons.org/publicdomain/zero/1.0/) applies to the data made available in this article, unless otherwise stated in a credit line to the data. 
T-cell epitope regions including T317K, E317K, Q318E, K321N, I322K, T322K, R322K, K324Q, I327L, G352N, S354P, R355K, N356D, Q357E, and E361A.

Conclusions: In this study, the results indicated a high conservation at the pfcsp gene. This may further contribute in understanding the genetic polymorphisms of P. falciparum prior to the deployment of the RTS,S vaccine in Sudan.

Keywords: Malaria, Plasmodium falciparum circumsporozoite protein (PfCSP), Vaccine, RTS,S, Hapd, N-terminal, central repeats, and C-terminal regions

\section{Background}

Malaria continues to be a major global health problem, particularly in Africa where Plasmodium falciparum is the most prevalent species. In 2019, the World Health Organization (WHO) estimated about 229 million cases and 409 thousand deaths worldwide, with 95\% of malaria occurrence to be reported from Africa [1]. In Sudan, malaria is a serious public health problem and over $75 \%$ of the country population at risk of the disease [1]. The global 2016-2030 technical strategy for malaria aims to achieve $90 \%$ reduction in malaria incidences by 2030 worldwide [2]. The systematic fight against malaria consist of enhanced surveillance and monitoring, vector control, case-detection and treatment in cooperation with improved diagnosis and treatment capacity [3] yet there are several rapidly developing challenges for this approach mainly including the emerging resistance for drugs and insecticides, and the limited resources and diagnostic capacity in the endemic countries. However, effective vaccine will offer a powerful alternative that might make a breakthrough in this fight [2].

Malaria vaccine has been recommended as one of the pillars for the successful malaria eliminate $[2,4]$. In the last two decades, a newly designed vaccine to encounter $P$. falciparum malaria infection was developed based on the $P$. falciparum circumsporozoite protein gene ( $p f c s p$ ) which secreted by the sporozoite stage [5]. The $p f c s p$ gene is known to be subdivided into 3 regions namely the $\mathrm{N}$-terminal region, the central repeats region, and the $\mathrm{C}$-terminal region. The $\mathrm{N}$-terminal region is located at the $5^{\prime}$ end of PfCSP, comprised of around 84 to 104 amino acids (aa) [6]. The $\mathrm{N}$-terminal region is also characterized by the presence of the KLKQP motif which is essential for the parasite entry to the hepatocytes. This along with the Th1R T-cell epitope which induces the antibody response in the human host [7]. For the C-terminal region which present at the $3^{\prime}$ end, it encompasses the polymorphic Th2R and Th3R T-cell sub-regions which are also been recognized by the human immune system [6]. Concerning the central repeats region, it is consisted of multiple motifs repeats of 4 aa. The NANP and the NVDP motifs were mostly seen among the different $P$. falciparum strains with NANP more frequent [8]. However, several other motifs were also reported from different geographical regions [9].

The currently used malaria vaccine, is comprised of the central repeat region and the T-cell epitope of the c-terminal region of the $P$. falciparum 3D7 together with hepatitis $B$ virus surface antigen (HBsAg) [10]. The deployment of the RTS,S vaccine took place in a few African countries including Ghana, Malawi, and Kenya [11]. The vaccine showed variable immunization duration course that might last for up to 1 year; hence a booster dose is recommended especially in areas of high malaria multiplicity and intensity [12].

In Sudan, malaria is serious public health problem and under certain circumstances it develops to an epidemic level [13]. The national malaria control programme is challenged by the emergence and rapid development of both, drugs and insecticide resistance, urging the need for alternative tools for the disease control such as an effective vaccine $[14,15]$. It occurs with variable severity onset and syndrome throughout the year, however, in some parts of the country malaria incidence is sporadic signifying the genetic diversity of the circulating $P$. falciparum and consequently promote the need to identify and characterize parasite populations that circulate in each specific region [14]. Therefore, this study is aimed at assessing the genetic diversity and population structure of the P. falciparum parasite in Khartoum state, Sudan, based on the $p f c s p$ gene in order to assess congruency with different PfCSP reference strains together with different Sudanese isolates [16]. Moreover such data help in assess opportunity for the RTS,S vaccine successful deployment and evaluate effectiveness of the vaccine and aid in developing Sudan RTS,S vaccine [11].

\section{Methods}

Study sites and Plasmodium falciparum isolates

Plasmodium falciparum isolates were collected from different health centres in Khartoum State between January and December 2019. Khartoum state is one of the eighteen states of Sudan and considered as the capital of Sudan. It covers around $22.142 \mathrm{~km}^{2}$ and populated with around 7.688 million in 2017 censuses. Khartoum state is comprised of 7 localities; Khartoum, Omdurman, Bahri, Gebel Aulia, Karari, Umbada, and East Nile (Fig. 1). During the study period, a total of 261 febrile patients were recruited. Malaria diagnosis was made by expert 


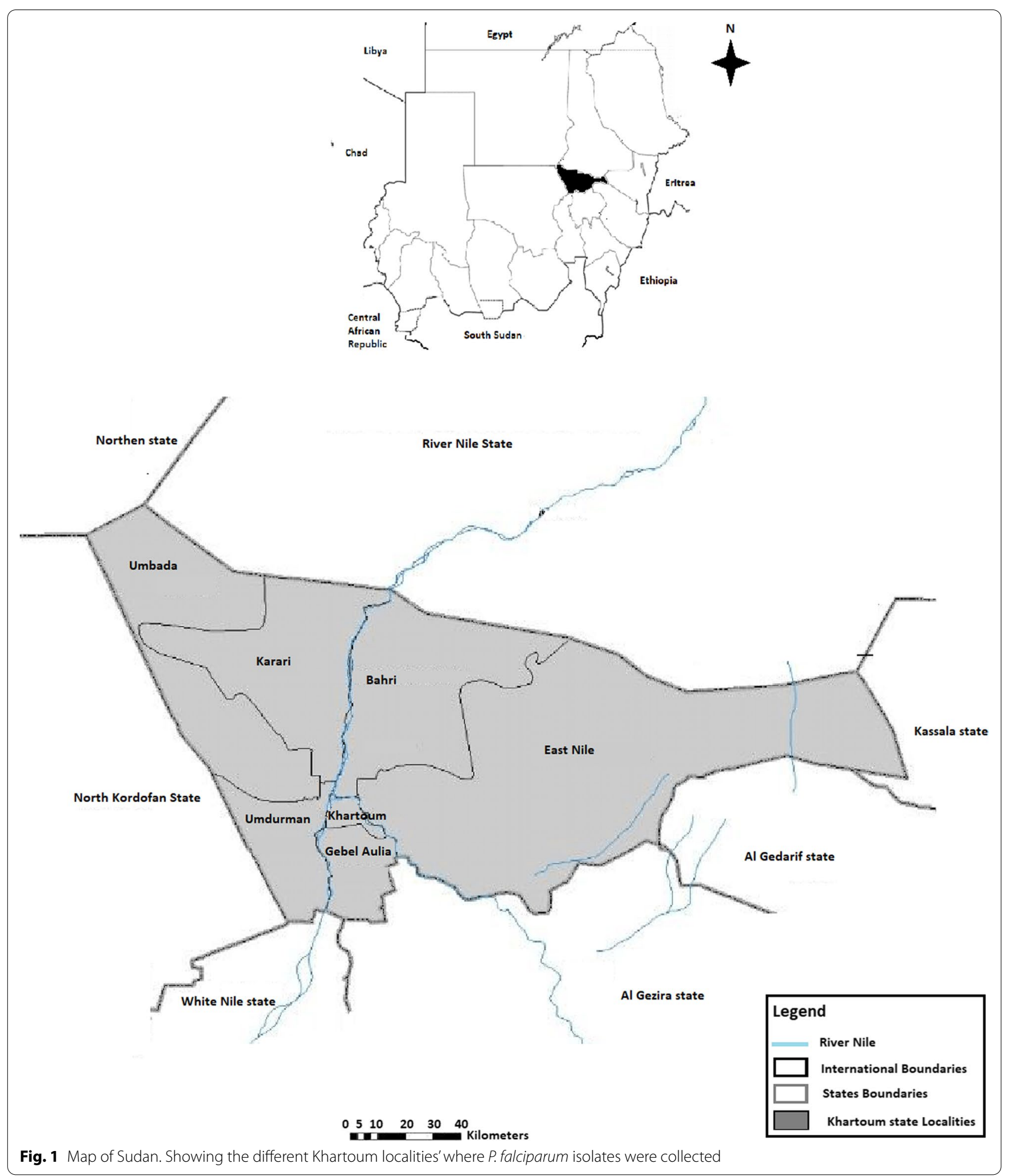


microscopists using Giemsa-stained thick and thin blood films. Prior to samples collection, patients or legal guardians of children less than 18 years old were offered a detailed description of the study, and if they have voluntary accept to enroll in the study, they were requested to sign an inform consent to include their samples and information in the study. Demographic and clinical data were collected. The Clinical phenotyping of malaria infection were made according to WHO guidelines [17]. The collected blood samples were preserved at $4{ }^{\circ} \mathrm{C}$ until molecular examination. All methods followed for DNA extraction, Molecular detection and clonal infection determination of Plasmodium species, amplification and sequences analysis of the $p f c s p$ gene were performed in accordance with the relevant guidelines and regulations.

\section{Molecular detection of Plasmodium species}

The total genomic DNA of the parasite was extracted from patients' blood samples using the QIAamp DNA Blood Mini Kit (Qiagen Inc. Germany). In order to confirm the microscopic results, all DNA samples were examined by the semi nested polymerase chain reaction (PCR) using the following primers; UNR: $5^{\prime}$ GAC GGT ATC TGA TCG TCT T 3', PLF: $5^{\prime}$ AGT GTG TAT CAA TCG AGT TT $3^{\prime}$, and HUF: $5^{\prime}$ GAG CCG CCT GGA TAC CG $3^{\prime}$ for the first PCR reaction and PLF primer and falciparum-R: $5^{\prime}$ AGT TCC CCT AGA ATA GTT ACA $3^{\prime}$, vivax-R: $5^{\prime}$ AGG ACT TCC AAG CCG AAG $3^{\prime}$, ovale-R: $5^{\prime}$ GCA TAA GGA ATG CAA AGA ACA G $3^{\prime}$, and malariae-R: $5^{\prime}$ GCC CTC CAA TTG CCT TCT $3^{\prime}$, for the second PCR reaction to confirm the presence of P. falciparum mono-infection. PCR reaction was made using the Maxime ${ }^{\mathrm{TM}} \mathrm{i}$-Taq PCR premix kit (iNtRON Biotechnology, South Korea) according to the manufacturer instructions. Primers concentrations and thermocycling conditions were adjusted according to Rubio et al. [18].

\section{Molecular assessment of $P$. falciparum multiple clonal infections}

In order to identify single clonal infection of the confirmed P. falciparum cases, the Merozoite Surface Protein 1 (msp 1 ) gene was amplified to exclude multiclonal infections. Nested PCR was done using the following primers; outer msp1-f: $5^{\prime}$ CTA GAA GCT TTA GAA GAT GCA GTA TTG $3^{\prime}$ and outer msp1-r: $5^{\prime}$ CTT AAA TAA TAT TCT AAT TCA AGT GGA TCA $3^{\prime}$. Following the first outer reaction clonal primers were used including MAD20-f: $5^{\prime}$ AAA TGA AGA AGA AAT TAC TAC AAA AGG TGC $3^{\prime}$, MAD20-r: 5' GCT TGC ATC AGC TGG AGG GCT TGC ACC AGA 3', RO33-f: 5' TAA AGG ATG GAG CAA ATA CTC AAG TTG TTG 3',
RO33-r: 5' CAT CTG AAG GAT TTG CAG CAC CTG GAG ATC $3^{\prime}$, K1-f: $5^{\prime}$ AAA TGA AGG AAC AAG TGG AAC AGC TGT TAC $3^{\prime}$, and K1-r: $5^{\prime}$ ATC TGA AGG ATT TGT ACG TCT TGA ATT ACC $3^{\prime}$. The primers concentrations per each reaction and the PCR amplification conditions used were according to Ntoumi et al. [19].

\section{Amplification of the pfcsp gene}

After the amplification of the msp 1 gene, $P$. falciparum were sorted out to exclude multiclonal infection since multiple clonal infections produce multiple products of $p f c s p$, which later give non-specific sequencing results. Genomic DNA of single clonal infections were further used for the amplification of $p f c s p$. The $p f c s p$ gene amplification was done as previously published by Zeeshan et al. [9]; using the following primers; outer PfCSP-f: $5^{\prime}$ TTA GCT ATT TTA TCT GTT TCT TC $3^{\prime}$ and outer PfCSP-r: $5^{\prime}$ TAA GGA ACA AGA AGG ATA ATA CC $3^{\prime}$, for the first PCR reaction. The second PCR reaction was made using the primers; nested nPfCSP-f: $5^{\prime}$ GAA ATG AAT TAT TAT GGG AAA CAG $3^{\prime}$ and nPfCSP-r: 5' GAA GGA TAA TAC CAT TAT TAA TCC $3^{\prime}$. PCR amplicons of the first run were used as a template for the second PCR. The amplified $p f c s p$ products were visualized using agarose gel electrophoresis in comparison to a 100 base pair DNA ladder. Amplified PfCSP PCR products were further purified from gel using gel extraction kits and instructions were followed according to the manufacturer's guidelines (New England Biolabs Inc, New England). Each amplicon was sent in replicate for sequencing by the Sanger dideoxy sequencing method using the primer SeqPfCSP: $5^{\prime}$ TGG GTC ATT TGG CAT ATT GTG $3^{\prime}$ using ABI3500 sequencer (Applied Biosystems SeqStudio, 3500 series) provided by Macrogen Inc. (The Netherlands).

\section{pfcsp sequence analysis}

The obtained sequences of the PfCSP amplicons were checked for the correctness of sequences reads and nucleotides base calling errors by aligning of the forward and the complementary reverse sequence using GENtle software (v1.9.4) [20]. Sequences were also trimmed at the primers sequences reads to reduce sequencing mismatching that occurs at the beginning of the sequencing process. Using the NCBI GenBank database, all worldwide previously published PfCSP sequences were included in the analysis of the comparison with the Sudanese sequences similarity and divergence (https://www.ncbi. nlm.nih.gov/nuccore). Local Sudanese sequences comparison and divergence were made on the N-terminal, C-terminal and the NANP repeat regions of the PfCSP gene. Reference sequences of the $p f c s p$ gene including the 
3D7 (XM_001351086) [21], NF54 (M22982.1) [22], 7G8 (AB121015.1), HB3 (AB121018.1), Dd2 (AB121017.1), MAD20 (AB121020.1), and RO33 (AB121021.1) [23], and the Wellcome strain (M15505.1) [24]. Using MEGA7 software, the nucleotide substitution model with the least Bayesian Information Criterion (BIC) score was considered as a best fit model to conduct the phylogenetic tree [25]. The phylogenetic tree was conduct to investigate the relationship of the Sudanese PfCSP sequences with the PfCSP reference sequences. Hasegawa-KishinoYano with Gamma distribution model $(\mathrm{HKY}+\mathrm{G}+\mathrm{I})$ was used for creating the tree [26]. Using the software DnaSP (v5.10) [27], nucleotides diversity parameters including the numbers of haplotypes (Hap), haplotypes diversity (Hapd), the average number of nucleotide differences between two sequences $(\mathrm{P})$, and the numbers of segregating sites $(\mathrm{S})$ were obtained. To investigate the genetic relationship of the Sudanese PfCSP sequences with the worldwide PfCSP sequences, and since the central repeat region is an immunodominant epitope of PfCSP, and it had been applied to the component of RTS,S malaria vaccine, the haplotypes network was constructed using the online tcsBU software [28] Natural selection theory was also tested on the C-terminal region of PfCSP using Fuand Li's D, Fuand Li's F statistics, and Tajima's D test using DnaSP $[29,30]$ to investigate the role of immunity in the initiation of sequence divergence at the $\mathrm{Th} 2$ and $\mathrm{Th} 3$ located at the C-terminal region.

\section{Statistical analysis}

Data analysis was made using the Statistical Package for Social Sciences (SPSS version 20). Chi-Square test was calculated to test the significance association between the msp1 gene and the presence of the insertion at the $\mathrm{N}$-terminal region, and the number of NANP repeats at the Central repeat region. A $p$ value $<0.05$ was considered a statistically significant.

\section{Results}

\section{Genotyping and assessment of $P$. falciparum multiple} clonal infections

The study included a total of 261 febrile patients consisted of 143 (54.8\%) male and 118 (45.2\%) females. All study participants have reported fever $>37.5{ }^{\circ} \mathrm{C}$. Based on the WHO classification, all patients were classified as mild uncomplicated malaria infection. The microscopic examinations revealed that a total 196 (75.1\%) patients were positive for $P$. falciparum infection whiles the remaining 65 (24.9\%) were harbouring co-infection of $P$. falciparum/Plasmodium vivax. Molecular analysis using the semi-nested PCR confirmed the presence of $23.9 \%$ (47/196) P. falciparum/P. vivax co-infections. Confirmed cases of mono-infections of $P$. falciparum were further amplified for the msp1 gene to exclude multiple allelic infections which will later affect the sequencing process of the $p f c s p$ gene. Out of the 149 P. falciparum monoinfections, msp 1 revealed the presence of $83(55.7 \%)$ multiple allelic infection which were excluded from the amplification process of the $p f c s p$ gene. The selected 66 (44.3\%) P. falciparum single allelic, mono-infections isolates were amplified for the $p f c s p$ gene. PCR amplification produced product sizes that vary in length from $1 \mathrm{~kb}$ to $1.2 \mathrm{~kb}$.

\section{Phylogenetic network of the Sudanese pfcsp and pfcsp reference sequences}

In comparison with the different PfCSP reference strains, the Sudanese isolates showed a considerable similarity with other African isolates. The phylogenetic analysis showed that most of the sequences clustered together forming a major 2 different clades consisting of the majority of the study isolates, also withing the two major clades, sequences divergence was noticed. The first clade consisted of 24 isolates of them 18 isolates were clustered together with a bootstrap value of $100 \%$ similarity and the other 6 isolates clustered together with a bootstrap value of 63 . For the second major clade, 18 isolates clustered together with a bootstrap value of $100 \%$ and showing slight similarity with the Wellcome and the HB3 reference strains. The remaining isolates were distributed forming 2 different smaller clades with a considerable similarity with the 3D7, MAD20, RO33, Dd2, 7G8, and the NF54 reference strains (Fig. 2).

\section{Genetic diversity in the N-terminal region of the Sudanese and global pfcsp}

The analysis of the $\mathrm{N}$-terminal region showed the presence of 2 different haplotypes; Hap01 included the 3D7 reference strain plus 19 isolates, and Hap02 included 47 isolates. The haplotype diversity score was $0.425 \pm 0.00727$. The low haplotype diversity among the Sudanese isolates indicates that the $\mathrm{N}$-terminal region is highly conserved. The 2 haplotypes were observed due to the amino acid polymorphism at A98G. The amino acid insertion "61NNNGDNGREGKDEDKRDGNN80" was detected in a total of 47 isolates representing Hap02. Whereas the KLKQP motif was conserved in all the isolates (Fig. 3).

\section{Genetic diversity in the central repeat region of the Sudanese pfcsp}

Based on the NANP and NVDP repeats of the central repeat region, a total of 11 haplotypes were seen with a Hapd of $0.779 \pm 0.00097$. Hap01 and Hap02 were the most frequent haplotypes; 24 isolates and 18 isolates, respectively, followed by haplotypes Hap03, Hap05, and Hap06 which consisted of 9, 7, and 2 isolates, 


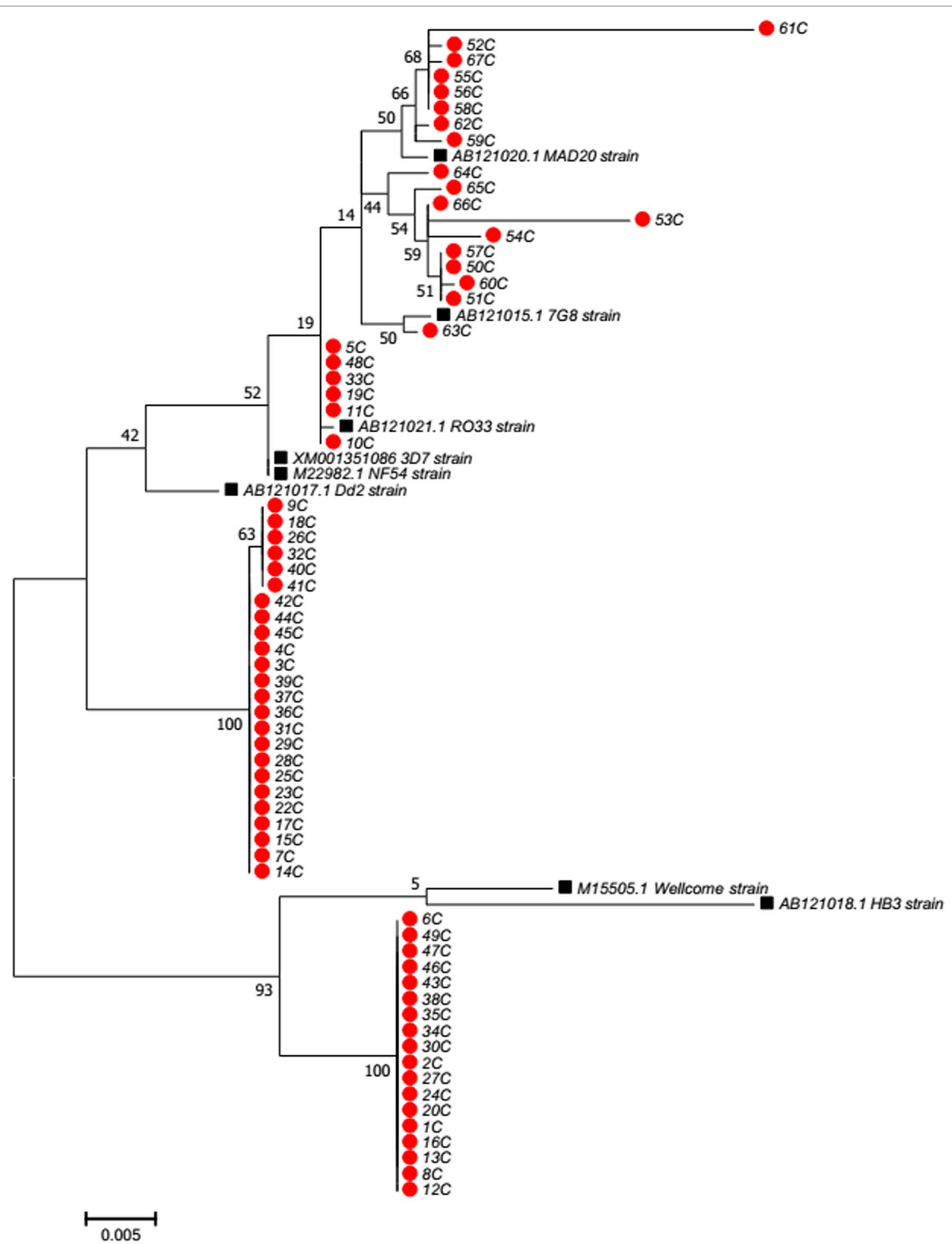

Fig. 2 Phylogenetic network of the complete Sudanese PfCSP sequences and the PfCSP reference sequences. Red circles correspond the Sudanese PfCSP sequences. Black boxes correspond the PfCSP reference sequences. 3D7 (XM_001351086), NF54 (M22982.1), 7G8 (AB121015.1), HB3 (AB121018.1), Dd2 (AB121017.1), MAD20 (AB121020.1), and RO33 (AB121021.1), and the Wellcome strain (M15505.1) 


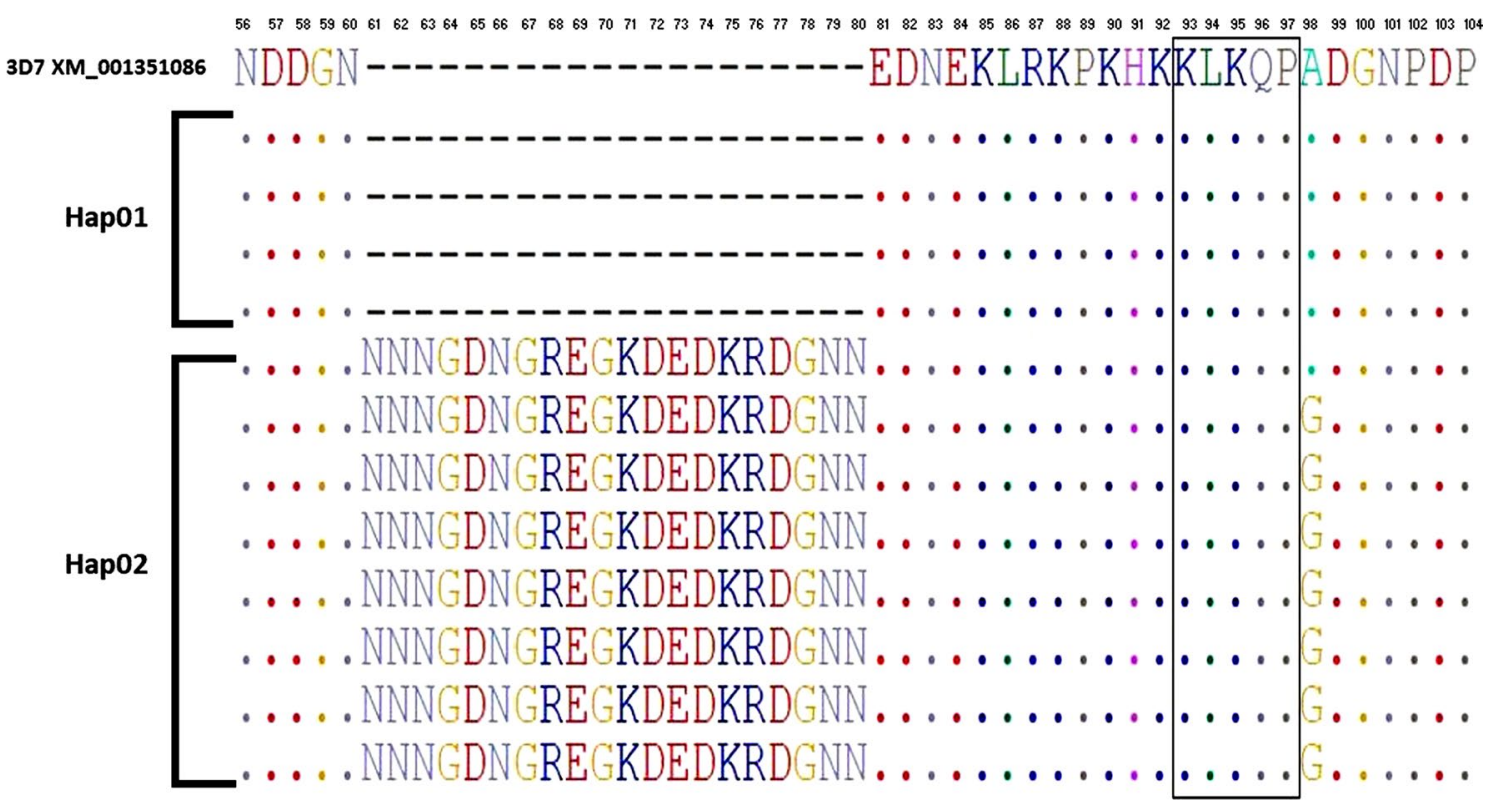

Fig. 3 Sequence diversity in the PfCSP N-terminal region of the Sudanese isolates. Comparison was made in relation to the P. falciparum reference sequence 3D7 (Accession No: XM_001351086). The dashes present the insertion NNNGDNGREGKDEDKRDGNN, which is present only in samples belonging to Hap02. Hap01 was consisted of 19 isolates, and Hap02 consisted of 47 isolates. Sequences shown are representative sequences to the total sequences investigated to present the insertion. KLKQP motif indicated with a rectangular is conserved through all the study isolates

\begin{tabular}{|c|c|c|c|}
\hline Haplotypes & 102 & Central repeat region & \\
\hline 3D7 & PDP 1 & 1212121211111111111112111111111111111111 NKNN & \\
\hline Hap01 & $\ldots 1$ & $1212121211111111111112111111111111111111 \ldots$ & 24 \\
\hline Hap02 & $\ldots 1$ & $1212121211111211121111111111111111111111 \ldots$ & 18 \\
\hline Hap03 & $\ldots 1$ & $1212121111111111111111111111111111111111 \ldots$ & 9 \\
\hline Hap04 & $\ldots 1$ & $1212121111111111111121111111111111111111 \ldots$ & 1 \\
\hline Hap05 & $\ldots 1$ & $121212111111111111112111111111111111111 \ldots \ldots$ & 7 \\
\hline Hap06 & $\ldots$ & $12121211111111111121111111111111111111-\ldots$ & 2 \\
\hline Hap07 & & $1211111121211111111211111111111111111111 \ldots$ & 1 \\
\hline Hap08 & $\ldots 1$ & $1212121111111111111121111111111111111111 \ldots$ & 1 \\
\hline Hap09 & $\ldots$ & $1212121111111111111121111111111111111--\ldots \ldots$ & 1 \\
\hline Hap10 & $\ldots 1$ & $121212111111111111221111111111111111111--\ldots$ & 1 \\
\hline Hap11 & $\ldots 1$ & $1212121111111111121111111111111111111--\ldots \ldots$ & 1 \\
\hline
\end{tabular}

Fig. 4 Sequence diversity in the central repeat region of the Sudanese PfCSP gene. Comparison was made in relation to the $P$. falciparum reference sequence 3D7 (Accession No: XM_001351086). 1 represents NANP motif, 2 represents NVDP motif. Of the 66 sequences investigated, numbers of isolates per each haplotype detected are indicated

correspondingly. Whereas the remaining haplotypes consisted of a single isolate per each. The numbers of NANP repeats at each haplotype were ranging from 33 to 37 repeats whereas NVDP repeats were ranging from 3 to 6 repeats. Accordingly, the total number of repeats per haplotype was ranging from 37 to 40 repeats (Fig. 4).
Association of $m s p-1$ genotyping with sequences diversities at the $\mathrm{N}$-terminal and the central repeat regions of the Sudanese pfcsp genes

Analysis of sequences diversity at the N-terminal and the central repeat regions of the PfCSP gene in relation with the different $m s p 1$ genotypes did not show any significant association between the different msp1 genotypes and the presence of insertion in the N-terminal 
Table 1 Association of MSP-1 genotyping with sequences diversities at the N-terminal and the central repeat regions of the Sudanese PfCSP genes

\begin{tabular}{|c|c|c|c|c|c|c|c|c|c|c|c|c|}
\hline \multirow[t]{2}{*}{ MSP-1 genotype } & \multirow[t]{2}{*}{ Sample origin } & \multicolumn{2}{|c|}{$\begin{array}{l}\text { Presence of } \\
\text { insertion in } \\
\text { the } N \text {-terminal } \\
\text { region of } \\
\text { PfCSP }\end{array}$} & \multirow[t]{2}{*}{ Total } & \multirow[t]{2}{*}{ P value } & \multicolumn{5}{|c|}{ Number of NANP motif repeats } & \multirow[t]{2}{*}{ Total } & \multirow[t]{2}{*}{$P$ value } \\
\hline & & Yes & No & & & 33 & 34 & 35 & 36 & 37 & & \\
\hline \multirow[t]{7}{*}{ MAD20 } & Khartoum & 6 & - & 6 & 0.428 & - & - & 6 & - & - & 6 & 0.787 \\
\hline & Bahri & 1 & - & 1 & & - & - & 1 & - & - & 1 & \\
\hline & East Nile & 1 & - & 1 & & - & - & 1 & - & - & 1 & \\
\hline & Gebel Aulia & 1 & 1 & 2 & & - & - & 2 & - & - & 2 & \\
\hline & Karari & 1 & - & 1 & & - & - & 1 & - & - & 1 & \\
\hline & Omdurman & 1 & - & 1 & & - & - & 1 & - & - & 1 & \\
\hline & Umbada & 1 & - & 1 & & - & - & 1 & - & - & 1 & \\
\hline \multirow[t]{6}{*}{ K1 } & Khartoum & 5 & 2 & 7 & 0.361 & - & 5 & 2 & - & - & 7 & 0.440 \\
\hline & Bahri & 2 & - & 2 & & - & - & 2 & - & - & 2 & \\
\hline & Gebel Aulia & 3 & 2 & 5 & & - & 3 & 2 & - & - & 5 & \\
\hline & Karari & 2 & - & 2 & & - & - & 2 & - & - & 2 & \\
\hline & Omdurman & 2 & 3 & 5 & & - & 2 & 2 & - & 1 & 5 & \\
\hline & Umbada & 3 & - & 3 & & - & 1 & 2 & - & - & 3 & \\
\hline \multirow[t]{7}{*}{ RO33 } & Khartoum & 4 & 6 & 10 & 0.358 & - & 4 & 2 & 2 & 2 & 10 & 0.763 \\
\hline & Bahri & - & - & 0 & & - & - & - & - & - & 0 & \\
\hline & East Nile & 5 & 1 & 6 & & 2 & 1 & 1 & - & 2 & 6 & \\
\hline & Gebel Aulia & 2 & 2 & 4 & & - & 1 & 1 & - & 2 & 4 & \\
\hline & Karari & 4 & 1 & 5 & & - & 3 & - & 1 & 1 & 5 & \\
\hline & Omdurman & 1 & - & 1 & & - & - & 1 & - & - & 1 & \\
\hline & Umbada & 1 & 2 & 3 & & 1 & - & 1 & - & 1 & 3 & \\
\hline Total & & 46 & 20 & 66 & & 3 & 20 & 31 & 3 & 9 & 66 & \\
\hline
\end{tabular}

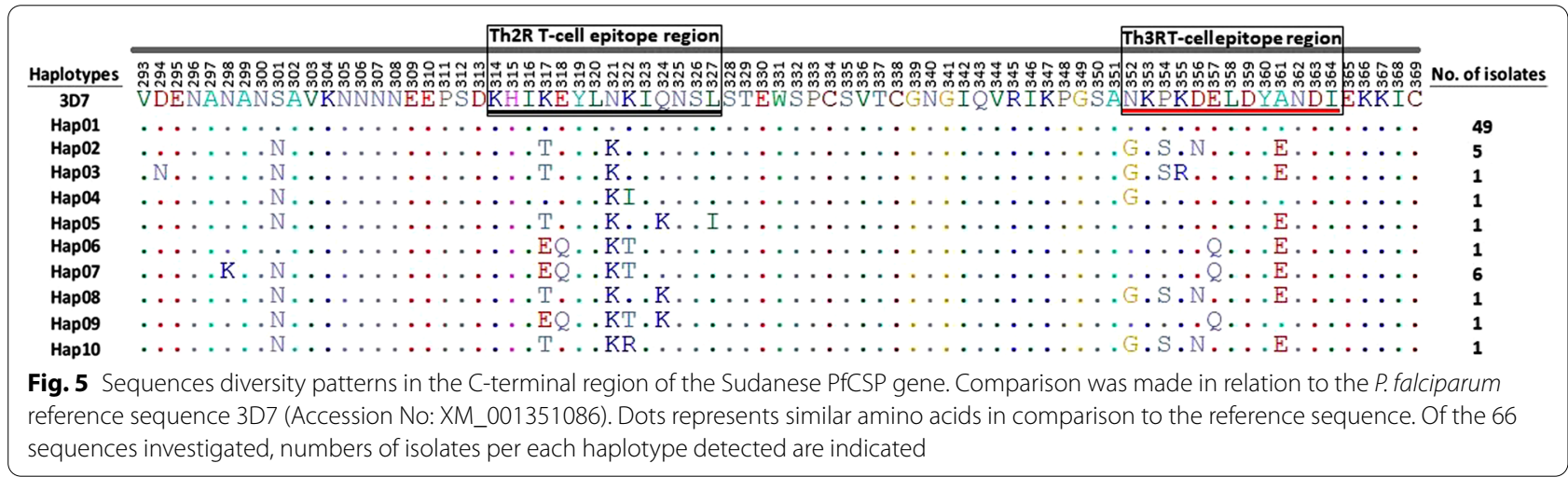

region nor the number of NANP motif repeats of the PfCSP gene, $p$ values $>0.05$ (Table 1 ).

\section{Diversity patterns in the C-terminal region of the Sudanese pfcsp}

The analysis of the genetic diversity in the C-terminal region of the Sudanese $p f c s p$ gene showed the presence of 10 different haplotypes with a haplotype diversity of $0.457 \pm 0.073$. Hap01 was the most frequent haplotype; 49 isolates. Hap02 was reported among 5 isolates, Hap07 was reported among 6 isolates. the remaining haplotypes were only represented by one isolate each (Fig. 5). Non-synonymous substitutions were seen in different amino acids in the $\mathrm{C}$-terminal region. 


\begin{tabular}{|c|c|}
\hline \multicolumn{2}{|c|}{ Groups } \\
\hline & Asembo Bay \\
\hline & Tanzania \\
\hline & Venezuela \\
\hline & Thailand \\
\hline & Sudan \\
\hline & Ghana \\
\hline & Brazil \\
\hline & PNG \\
\hline & Philippines \\
\hline & Solomon Islands \\
\hline & India \\
\hline & Cameroon \\
\hline & Gambia \\
\hline & Myanmar \\
\hline & Iran \\
\hline & Vanuatu \\
\hline
\end{tabular}

Fig. 6 Haplotype network analysis and sequences diversity patterns in the C-terminal region of the Sudanese and global pfcsp gene. Haplotypes numbers of the main haplotypes are indicated at each node. The number of sequences of the Sudanese isolates are indicated in brackets next to their representative haplotype
D294N, K298N, and N301S, were seen in Hap03, Hap07, and all the haplotypes except for Hap01 and Hap06, respectively. Several non-synonymous amino acids changes were also seen at the Th2R and the Th3R T-cell epitope regions including T317K, E317K, Q318E, K321N, I322K, T322K, R322K, K324Q, I327L, G352N, S354P, R355K, N356D, Q357E, and E361A (Fig. 5). The analysis of the C-terminal nucleotide diversity was $0.00929 \pm 0.00150$, and an average number of nucleotide differences of 3.51199 was observed. Neutrality testing indices to investigate the null hypothesis of natural selection using $\mathrm{Fu}$ and Li's $\mathrm{D}^{*}, \mathrm{Fu}$ and Li's $\mathrm{F}^{*}$, and Tajima's D tests were $-0.40167,-0.45855$, and -0.35629 . All tests were statistically not significant, $\mathrm{P}>0.10$.

\section{Haplotype network analysis and diversity patterns} in the C-terminal region of the Sudanese and global PfCSP Based on the C-terminal region, the analysis of the haplotype network of the Sudanese isolates and the global published C-terminal region of the PfCSP the number of haplotypes detected was 69 different haplotypes with a haplotype diversity of $0.8651 \pm 0.008$. Hap11 was the most frequent haplotype, followed by Hap43, Hap12. The haplotype that includes the 3D7 reference strain was considered Hap01. Hap01 was including most of the studied Sudanese isolates; 48 isolates out of 66 isolates. The remaining isolates were distributed into Hap02 (5 isolates), Hap03 (1), Hap04 (1), Hap05 (1), Hap06 (1), Hap07 (6), Hap08 (1) Hap09 (1), and Hap10 (1) (Fig. 6).

\section{Discussion}

In Khartoum State, $P$. falciparum is accounted for up to $90 \%$ of malaria infections, while $10 \%$ are acknowledged to be caused by $P$. vivax. Investigating the genetic diversity of the Sudanese $P$. falciparum is extremely important to further warrant the deployment of the RTS,S malaria vaccine [16]. The aim of this study was to investigate the genetic diversity of $p f c s p$ gene in Sudan. In this study, the investigated $p f c s p$ gene showed a high conversation at the $\mathrm{N}$-terminal region when compared to the 3D7 reference strain. Although, a higher conservation is reported in this study in which only 2 haplotypes were detected 
compared to previous studies which could be attributed to the difference in location which was reported from different regions of Sudan and also the sample size included, where they only investigated 21 P. falciparum $\mathrm{N}$-terminal region sequences, and specifically only 4 sequences from Khartoum were investigated [16]. Also, the presence of the other unique polymorphisms can be certain to each specific location depending on the circulating $P$. falciparum strain. The (NNGDNGREGKDEDKRDGNN) insertion which was not reported previously could be mainly due to the small sample size been investigated which led to the misdetection of this insertion previously which is noticeable by the small frequency of the insertion reported in this study. Meanwhile, this insertion has been detected in several other geographical regions, such as Myanmar [8].

Although, the functional role of this insertion when it's present or absent is limited investigated and not fully understood yet. Although analysis of the different msp 1 genotypes with sequences diversities at the $\mathrm{N}$-terminal region of the Sudanese $p f c s p$ gene did not reveal any association for the presence of the insertion. therefore the presence of the insertion could be explained in terms of different parasite strains, geographical or environmental adaptation of the parasite, or it might be present as a form of parasite adaptation to the host immune response which is mainly targeted at the Th1R T-cell epitope present at the $\mathrm{N}$-terminal region [7]. Accordingly, the Th1R $\mathrm{T}$-cell epitope at the $\mathrm{N}$-terminal region is showing relatively high polymorphisms, this can be explained as a role of parasite adaptation to host immune system. This was previously investigated by Conway et al. when a monoclonal antibody anchored to the linear T-cell epitope "81EDNEKLRKPKH91" which is located before the KLKQP motif which is responsible for sporozoite entry to the hepatocytes [31]. The monoclonal antibody effectively neutralized the infectivity of the sporozoite in-vivo [31]. Also, Bongfen et al. demonstrated that the development of polypeptides that flank the $\mathrm{N}$-terminal region played a significant role in the initiation of inhibitory antibodies production to prevent sporozoite invasion to the hepatocytes [32]. Thus, supporting the hypothesis that the $\mathrm{N}$-terminal region can play an important role as a vaccine candidate which is aided by the high conservation rate [31].

This study has detected a high haplotype diversity at the central repeat region, this in turn has highlighted the genetic diversity of this region is mainly sustained by balancing natural selection due to the effect of human immune response [31]. This also indicated by the negative, statistically insignificant values of $\mathrm{Fu}$ and Li's $\mathrm{D}^{*}$, $\mathrm{Fu}$ and Li's $\mathrm{F}^{*}$, and Tajima's D tests. However, bottleneck effect and population expansion are both scenarios cannot be neglected completely. Interestingly, as the difference in the NANP and NVDP repeats controls the difference in the $p f c s p$ gene length, the numbers of NANP repeats detected among this studied isolates were lower than previously reported by Lê et al. [8] and Zeeshan et al. [9], where higher numbers of NANP repeats was detected as well as several unique repeat motifs including NANS, NTNP, NVVP, NAHP, NAKP, NVNP, NAIP, NANL, NPNP, NVAD, NADP, SANP, and KANP. However, this variation in the central repeat region requires more research to understand the effect of these variation in terms of parasite genetic signature and host immune response. Previously, the central repeat region has been found to affect the stability of the $p f c s p$ gene, which is suggested to be a copy-number dependent and further growths by the increase in the number of repeats [33]. The Sudanese isolates showed a relatively low numbers of repeats that ranged from 37 to 40 repeats similar to the African and South American PfCSP; 36 to 37 repeats, while considerably lower than the Asian PfCSP; 40 to 43 repeats [8]. This indicates that the difference in the numbers of the tetrapeptide repeats at the central repeat region could be attributed to geographical distribution, host (human and/or mosquito vectors) population genetic differences, or the different selection pressure imposed by the different case management protocols/drugs in use that resulted in form of co-evolution response to maintain parasite stability at specific origin. Also, the central repeat region is important in the RTS,S malaria vaccine. The RTS,S consisted of 19 tetrapeptide repeats of the NANP repeat [34]. However, still no reports indicated that the different repeats number can or may affect the effectiveness of the RTS,S vaccine (see Fig. 6).

Investigating the genetic diversity of the $\mathrm{C}$-terminal region of the Sudanese $p c s p$ gene showed a relatively low genetic diversity expressed by the presence of only 10 haplotypes, with Hap01 to be the most frequent; 49 isolates, and it has two insertions; the "KHIEKEYLNKIQNSL" in the Th2R and "NKPKDELDYANDI" in the Th3R regions T-cell epitopes. This haplotype has been also prevalent among other African PfCSP isolates, this is further explained by the haplotype network where most of the Sudanese isolates clustered with the Ghanaian isolates. The low haplotype diversity detected among the Sudanese PfCSP isolates indicates a low level of genetic diversity. Since the RTS,S malaria vaccine is composed of the $\mathrm{C}$-terminal $\mathrm{T}$-cell epitope, this region can be very important in terms of designing regional specific vaccines based on identifying the circulating P. falciparum strains in that specific region [34].

The analysis of the global C-terminal region haplotype network indicated a unique clustering of the Asian 
isolates in a relatively separated haplotypes from the African and the South American isolates. This has been also discussed previously by Lê et al., where African isolates presented with branched and complex patterns of haplotypes [8]. This also supports the suggestion that African PfCSP is more diverse than the PfCSP of the other geographical regions.

Since the deployment of the RTS,S malaria vaccine is mainly controlled by understanding and identifying the genetic diversity of the circulating $P$. falciparum strains in a certain geographical region [11]. RTS,S vaccine trials showed a significant effect in reducing malaria incidence in many African countries including Gambia, Mozambique, Ghana, Tanzania, Gabon, and Kenya [35-37]. Therefore, this study findings are showing the higher prevalence of a haplotype, which is more similar to the 3D7 reference strain and also worth mentioning that the current RTS,S is primarily constructed from the PfCSP of the 3D7 strain, the deployment of the RTS,S malaria vaccine clinical trials can be effective in reducing malaria incidence in Sudan.

Although, this study investigated the genetic diversity of $P$. falciparum pfcsp gene in Sudan, which is currently part of the RTS,S malaria vaccine formulation. However, despite the importance of mapping genetic diversity, the results are still at the level of simple gene sequence observations for the identification of sequence changes which might be validated for what concerns their impact on vaccine efficacy. The effects on parasite fitness are neither tested at the cell nor biochemical levels. Therefore, further experiments to establish how these changes impact parasite and/or host biology.

\section{Conclusion}

This study is the first study to investigate the genetic diversity of $P$. falciparum in Sudan based on studying the complete $p f c s p$ gene. This study was limited to investigating the genetic diversity in specific region in Sudan; Khartoum. Therefore, further studies are warranted to explore the genetic diversity of PfCSP in the whole country since Sudan is known with its large geographical space that has different environmental conditions and endemicity situations that might influence the development of different adaptive evolutionary traits among the parasite population increasing its genetic diversity. Also, there are potential evolutionary consequences could not be observed in this study samples were co-infections nor multiclonal infections were investigated. The combination of these factors might impact the effectiveness of the RTS,S vaccine if been deployed in Sudan or areas with similar scenario, that why a more comprehensive study with a larger sample size that collected from different regions is highly recommended. The $p f c s p$ gene is considered a core element of the RST,S malaria vaccine that currently been used, nevertheless, the genetic diversity in the $p f c s p$ gene among the different regions $P$. falciparum strains may affect the efficacy of the RTS,S vaccine. In this study, the analysis of the genetic diversity of the PfCSP population structure indicated that the $p f c s p$ gene in Sudan is highly conserved with few genetic variations reported at the central repeat region and the C-terminal region. Meanwhile, the limited diversity reported at the $\mathrm{N}$-terminal region postulates the usefulness of this region in the development of new vaccine candidate.

\section{Abbreviations \\ Hap: Haplotype; Hapd: Haplotype diversity; Msp1: Merozoite Surface Protein 1; $\mathrm{P}$ : Average number of nucleotide differences; PfCSP: Circumsporozoite surface protein of Plasmodium falciparum; S: Segregating sites.}

\section{Acknowledgements}

We are grateful to all individuals who agreed to participate in this study. NSM recipient of a partial fund from the Royal Society of Tropical Medicine and Hygiene to conduct the study (RSTMH Small Grant round 2019).

\section{Authors' contributions}

NSM, HA, and AA: Study designing. HA, AEA, ARE, MSA, and EES: Performed experiments. NSM, LTE, OA, YOM, AA, and RAO: Data analysis and manuscript preparation: Field work support: NSM, HA, AM, LTE, YA, and MABE. Manuscript review and data analysis: NSM, EES, AA, and RAO. MA: Created the study map. NSM and MA: Revision of manuscript and data analysis. All authors have read and approved the final manuscript.

\section{Funding}

Not applicable.

\section{Availability of data and materials}

All datasets generated for this study are included in the manuscript.

Sequences generated in this study are available at the NCBI GenBank database under the accession numbers; MW554526-MW554591.

\section{Declarations}

\section{Ethics approval and consent to participate}

Written consents were obtained prior to data and samples collection from all the study participants following being well informed about the study and potential risk and volunteering to participate, while written consent was taken from parents or legal guardians in case of children. Ethical approval for conducting the study was obtained from the National University Research Ethics Committee, National University-Sudan (NU-REC13).

\section{Consent for publication}

Not applicable.

\section{Competing interests}

The authors declare that they have no competing interests.

\footnotetext{
Author details

${ }^{1}$ Department of Parasitology and Medical Entomology, Tropical Medicine Research Institute, National Centre for Research, Khartoum, Sudan. ${ }^{2}$ Molecular Biology Unit, Sirius Training and Research Centre, Khartoum, Sudan. ${ }^{3}$ El-Raqi Hospital, National University, Khartoum, Sudan. ${ }^{4}$ Health Emergencies and Epidemics Control General Directorate, Sudan Federal Ministry of Health, Khartoum, Sudan. ${ }^{5}$ Faculty of Medicine, EL-Neelain University, Khartoum, Sudan. ${ }^{6}$ Department of the Integrated Vector Management (IVM), Federal Ministry of Health, Khartoum, Sudan. ${ }^{7}$ Mycetoma Research Center, University of Khartoum, Khartoum, Sudan. ${ }^{8}$ Pediatric Epidemiology, Clinic and Polyclinic for Child and Adolescent Medicine, Medical Faculty, University of Leipzig, Leipzig, Germany.
} 
Received: 13 July 2021 Accepted: 1 November 2021

Published online: 10 November 2021

\section{References}

1. WHO. World malaria report. 20 years of global progress and challenges. Geneva:World Health Organization; 2020. p. 2020.

2. WHO. Global technical strategy for malaria 2016-2030. Geneva: World Health Organization; 2015.

3. Shretta R, Liu J, Cotter C, Cohen J, Dolenz C, Makomva K, et al. Malaria elimination and eradication. In: Holmes KK, Bertozzi S, Blaoom BR, Jha P, editors., et al., Major infectious diseases. 3rd ed. Washington: The International Bankk for Recosntruction and Development/The World Bank; 2017.

4. WHO recommends groundbreaking malaria vaccine for children at risk. Historic RTS,S/ASO1 recommendation can reinvigorate the fight against malaria. https:/www.who.int/news/item/06-10-2021-who-recommends-groundbrea king-malaria-vaccine-for-children-at-risk. Accessed 10 Oct 2021.

5. Stoute JA, Slaoui M, Heppner DG, Momin P, Kester KE, Desmons P, Wellde BT, et al. A preliminary evaluation of a recombinant circumsporozoite protein vaccine against Plasmodium falciparum malaria. N Engl J Med. 1997;336:86-91.

6. Calvo-Calle JM, De Oliveira G, Clavijo P, Maracic M, Tam J, Lu Y, et al. Immunogenicity of multiple antigen peptides containing $B$ and non-repeat $T$ cell epitopes of the circumsporozoite protein of Plasmodium falciparum. J Immunol. 1993;150:1403-12.

7. Enea V, Ellis J, Zavala F, Arnot DE, Asavanich A, Masuda A, et al. DNA cloning of Plasmodium falciparum circumsporozoite gene: amino acid sequence of repetitive epitope. Science. 1984;225:628-30.

8. Lê HG, Kang J-M, Moe M, Jun H, Thái TL, Lee J, et al. Genetic polymorphism and natural selection of circumsporozoite surface protein in Plasmodium falciparum field isolates from Myanmar. Malar J. 2018;17:361.

9. Zeeshan M, Alam MT, Vinayak S, Bora H, Tyagi RK, Alam MS, et al. Genetic variation in the Plasmodium falciparum circumsporozoite protein in India and its relevance to RTS, S malaria vaccine. PLoS ONE. 2012;7: e43430.

10. Rutgers T, Gordon D, Gathoye AM, Hollingdale M, Hockmeyer W, Rosenberg $M$, et al. Hepatitis B surface antigen as carrier matrix for the repetitive epitope of the circumsporozoite protein of Plasmodium falciparum. Nat Biotechnol. 1988;6:1065-70.

11. Alonso PL, Sacarlal J, Aponte J, Leach A, Macete E, Milman J, et al. Efficacy of the RTS, S/AS02A vaccine against Plasmodium falciparum infection and disease in young African children: randomised controlled trial. Lancet. 2004:364:1411-20.

12. Penny MA, Galactionova K, Tarantino M, Tanner M, Smith TA. The public health impact of malaria vaccine RTS, S in malaria endemic Africa: country-specific predictions using 18 month follow-up Phase III data and simulation models. BMC Med. 2015;13:170

13. Aradaib IE, Erickson BR, Karsany MS, Khristova ML, Elageb RM, Mohamed ME, et al. Multiple Crimean-Congo hemorrhagic fever virus strains are associated with disease outbreaks in Sudan, 2008-2009. PLoS Negl Trop Dis. 2011;5: e1159.

14. Ageep TB, Damiens D, Alsharif B, Ahmed A, Salih EH, Ahmed FT, et al. Participation of irradiated Anopheles arabiensis males in swarms following field release in Sudan. Malar J. 2014;13:484

15. Mohamed NS, Abdelbagi H, Osman HA, Ahmed AE, Yousif AM, Edris YB, et al. A snapshot of Plasmodium falciparum malaria drug resistance markers in Sudan: a pilot study. BMC Res Notes. 2020;13:512.

16. Mohamed NS, Albsheer MMA, Abdelbagi H, Siddig EE, Mohamed MA, Ahmed $\mathrm{AE}$, et al. Genetic polymorphism of the N-terminal region in circumsporozoite surface protein of Plasmodium falciparum field isolates from Sudan. Malar J. 2019;18:333.

17. WHO. Guidelines for the treatment of malaria. Geneva: World Health Organization; 2015.
18. Rubio J, Benito A, Berzosa P, Roche J, Puente S, Subirats M, et al. Usefulness of seminested multiplex PCR in surveillance of imported malaria in Spain. J Clin Microbiol. 1999;37:3260-4.

19. Ntoumi F, Ngoundou-Landji J, Lekoulou F, Luty A, Deloron P, Ringwald P. Site-based study on polymorphism of Plasmodium falciparum MSP-1 and MSP-2 genes in isolates from two villages in Central Africa. Parassitologia. 2000;42:197-203.

20. Manske M. GENtle, a free multi-purpose molecular biology tool. Universität zu Köln. 2006.

21. Hall N, Pain A, Berriman M, Churcher C, Harris B, Harris D, et al. Sequence of Plasmodium falciparum chromosomes 1, 3-9 and 13. Nature. 2002:419:527-31.

22. Caspers P, Gentz R, Matile H, Pink JR, Sinigaglia F. The circumsporozoite protein gene from NF54, a Plasmodium falciparum isolate used in malaria vaccine trials. Mol Biochem Parasitol. 1989;35:185-9.

23. Tanabe K, Sakihama N, Hattori T, Ranford-Cartwright L, Goldman I, Escalante AA, et al. Genetic distance in housekeeping genes between Plasmodium falciparum and Plasmodium reichenowi and within P. falciparum. J Mol Evol. 2004;59:687-94

24. Lockyer MJ, Schwarz RT. Strain variation in the circumsporozoite protein gene of Plasmodium falciparum. Mol Biochem Parasitol. 1987;22:101-8.

25. Kumar S, Stecher G, Tamura K. MEGA7: molecular evolutionary genetics analysis version 7.0 for bigger datasets. Mol Biol Evol. 2016;33:1870-4.

26. Kishino $\mathrm{H}$, Hasegawa M. Evaluation of the maximum likelihood estimate of the evolutionary tree topologies from DNA sequence data, and the branching order in Hominoidea. J Mol Evol. 1989;29:170-9.

27. Librado P, Rozas J. DnaSP v5: a software for comprehensive analysis of DNA polymorphism data. Bioinformatics. 2009;25:1451-2.

28. Múrias dos Santos A, Cabezas MP, Tavares Al, Xavier R, Branco M. tcsBU: a tool to extend TCS network layout and visualization. Bioinformatics. 2016;32:627-8.

29. Fu Y-X, LiW-H. Statistical tests of neutrality of mutations. Genetics. 1993;133:693-709.

30. Tajima F. Statistical method for testing the neutral mutation hypothesis by DNA polymorphism. Genetics. 1989;123:585-95.

31. Conway DJ. Natural selection on polymorphic malaria antigens and the search for a vaccine. Parasitol Today. 1997;13:26-9.

32. Bongfen SE, Ntsama PM, Offner S, Smith T, Felger I, Tanner M, et al. The N-terminal domain of Plasmodium falciparum circumsporozoite protein represents a target of protective immunity. Vaccine. 2009;27:328-35.

33. Escalante AA, Grebert HM, Isea R, Goldman IF, Basco L, Magris M, et al. A study of genetic diversity in the gene encoding the circumsporozoite protein (CSP) of Plasmodium falciparum from different transmission areas-XVI. Asembo Bay Cohort Project. Mol Biochem Parasitol. 2002;125:83-90.

34. Garçon N, Heppner DG, Cohen J. Development of RTS, S/ASO2: a purified subunit-based malaria vaccine candidate formulated with a novel adjuvant. Expert Rev Vaccines. 2003;2:231-8.

35. Bojang KA, Milligan PJ, Pinder M, Vigneron L, Alloueche A, Kester KE, et al. Efficacy of RTS, S/ASO2 malaria vaccine against Plasmodium falciparum infection in semi-immune adult men in The Gambia: a randomised trial. Lancet. 2001;358:1927-34.

36. Aponte JJ, Aide P, Renom M, Mandomando I, Bassat Q, Sacarlal J, et al. Safety of the RTS, S/ASO2D candidate malaria vaccine in infants living in a highly endemic area of Mozambique: a double blind randomised controlled phase I/ Ilb trial. Lancet. 2007:370:1543-51.

37. Asante KP, Abdulla S, Agnandji S, Lyimo J, Vekemans J, Soulanoudjingar S, et al. Safety and efficacy of the RTS, S/AS01E candidate malaria vaccine given with expanded-programme-on-immunisation vaccines: 19 month follow-up of a randomised, open-label, phase 2 trial. Lancet Infect Dis. 2011;11:741-9.

\section{Publisher's Note}

Springer Nature remains neutral with regard to jurisdictional claims in published maps and institutional affiliations. 